Louisiana State University

LSU Digital Commons

$12-7-2010$

\title{
Estimate of the uncertainties in the relative risk of secondary malignant neoplasms following proton therapy and intensity- modulated photon therapy
}

\author{
Jonas D. Fontenot \\ Mary Bird Perkins Cancer Center \\ Charles Bloch \\ Washington University in St. Louis \\ David Followill \\ University of Texas MD Anderson Cancer Center \\ Uwe Titt \\ University of Texas MD Anderson Cancer Center \\ Wayne D. Newhauser \\ University of Texas MD Anderson Cancer Center
}

Follow this and additional works at: https://digitalcommons.Isu.edu/physics_astronomy_pubs

\section{Recommended Citation}

Fontenot, J., Bloch, C., Followill, D., Titt, U., \& Newhauser, W. (2010). Estimate of the uncertainties in the relative risk of secondary malignant neoplasms following proton therapy and intensity-modulated photon therapy. Physics in Medicine and Biology, 55 (23), 6987-6998. https://doi.org/10.1088/0031-9155/55/23/ S02

This Article is brought to you for free and open access by the Department of Physics \& Astronomy at LSU Digital Commons. It has been accepted for inclusion in Faculty Publications by an authorized administrator of LSU Digital Commons. For more information, please contact ir@lsu.edu. 


\title{
Estimate of the uncertainties in the relative risk of secondary malignant neoplasms following proton therapy and intensity- modulated photon therapy
}

\author{
Jonas D Fontenot ${ }^{1,4}$, Charles Bloch ${ }^{2}$, David Followill ${ }^{3}$, Uwe Titt $^{3}$, and Wayne D Newhauser ${ }^{3}$ \\ ${ }^{1}$ Department of Medical Physics, Mary Bird Perkins Cancer Center, Baton Rouge, LA, USA \\ ${ }^{2}$ Department of Radiation Oncology, Washington University, Saint Louis, MO, USA \\ ${ }^{3}$ Department of Radiation Physics, Unit 94, The University of Texas M D Anderson Cancer \\ Center, 1515 Holcombe Blvd, Houston, TX, USA
}

\section{Abstract}

Theoretical calculations have shown that proton therapy can reduce the incidence of radiationinduced secondary malignant neoplasms (SMN) compared with photon therapy for patients with prostate cancer. However, the uncertainties associated with calculations of SMN risk had not been assessed. The objective of this study was to quantify the uncertainties in projected risks of secondary cancer following contemporary proton and photon radiotherapies for prostate cancer. We performed a rigorous propagation of errors and several sensitivity tests to estimate the uncertainty in the ratio of relative risk ( $R R R$ ) due to the largest contributors to the uncertainty: the radiation weighting factor for neutrons, the dose-response model for radiation carcinogenesis and interpatient variations in absorbed dose. The interval of values for the radiation weighting factor for neutrons and the dose-response model were derived from the literature, while interpatient variations in absorbed dose were taken from actual patient data. The influence of each parameter on a baseline $R R R$ value was quantified. Our analysis revealed that the calculated $R R R$ was insensitive to the largest contributors to the uncertainty. Uncertainties in the radiation weighting factor for neutrons, the shape of the dose-risk model and interpatient variations in therapeutic and stray doses introduced a total uncertainty of $33 \%$ to the baseline $R R R$ calculation.

\section{Introduction}

The advancement of technologies in radiation therapy has accelerated rapidly in recent decades. Newer treatment planning and beam delivery technologies have enabled clinicians to escalate and conform doses to tumor volumes while simultaneously reducing radiation exposures to normal tissues. Normal tissue sparing is of increasing importance because cancers are being diagnosed at earlier ages and survival rates for primary cancers are increasing. For example, 5 year disease-specific survival rates for patients with prostate cancer are greater than 99\% (SEER 2007). However, epidemiological studies have

(C) 2010 Institute of Physics and Engineering in Medicine

${ }^{4}$ Author to whom any correspondence should be addressed. 
established that radiation therapy is risk factor for secondary malignant neoplasms (SMN) (Brenner et al 2000). The combination of these factors suggests that increasing numbers of patients treated for cancer will live long enough following treatment for a SMN to develop.

Ideally, the controversy surrounding the risk for developing a SMN following contemporary external-beam radiotherapies would be resolved with randomized patient outcome studies that include high-quality dose reconstructions and a 10 year or longer mean follow-up time. However, the inherent length of such studies limits their feasibility as well as their relevance to the latest treatment technologies. To overcome this problem, several investigations have instead focused on predicting the risk of SMN (Fontenot et al 2009b, Newhauser et al 2009c, Taddei et al 2008, 2009, Schneider et al 2006, 2007, 2008, Zacharatou Jarlskog et al 2008). In this approach, dose-response models from the literature have been combined with new dosimetric data to predict the risk of SMN incidence and mortality. The uncertainties of the risk projections calculated in this manner depend on many factors, including the endpoint (cancer incidence versus mortality), the type of risk (relative risk versus absolute risk), the accuracy of the therapeutic and stray radiation dose distributions, the relative biological effectiveness (RBE) of the radiation for carcinogenesis, the radiation type (e.g. photons versus protons), details of the beam delivery (e.g. scanned versus scattered proton beams), and the age and gender of the patient. For example, Newhauser et al (2009c) recently estimated the risk of SMN incidence for a pediatric patient receiving proton therapy for craniospinal irradiation, examining the impact of neutron RBE, scanned versus scattered proton beams and other factors on the risk analysis. More recently, Fontenot et al (2009b) reported on relative risk projections for patients receiving proton versus photon radiation therapies for cancer of the prostate. Although that work included a limited discussion of the corresponding uncertainties, the influence of the largest sources of uncertainty on the risk calculations was not examined. Namely, itwas unknown how the results of that studywould be affected by uncertainties in the dose-responsemodel for radiation carcinogenesis, uncertainties in the radiation weighting factor for neutrons, and interpatient variations in absorbed dose.

The objective of this study was to quantify the uncertainties in projected risks of SMN following contemporary proton and photon radiotherapies for cancer of the prostate. In particular, we performed a rigorous propagation of errors and several sensitivity tests to estimate the uncertainty in the ratio of relative risk $(R R R)$ following passively scattered proton therapy to that of intensity-modulated photon radiotherapy (IMRT).

\section{Methods and materials}

\subsection{Baseline RRR calculation}

Our previously reported methods for determining SMN risk in patients undergoing radiation therapy for early-stage prostate cancer (Fontenot et al 2009b) are summarized here. In brief, a two-field, parallel-opposed proton therapy plan and a seven-field $6 \mathrm{MV}$ photon IMRT plan were developed in accordance with clinical protocol at our institution. Normal tissue doses from the therapeutic beam were calculated by the treatment planning software (Eclipse, Varian Medical Systems, Palo Alto, CA). Stray doses from proton therapy treatments (e.g. neutrons) were determined fromMonte Carlo simulations (Newhauser et al 2009b, Fontenot 
et al 2008), while stray doses from IMRT (e.g. leakage) were determined from available data (Howell et al 2006, Kry et al 2005). Neutron doses emanating from proton treatment were weighted using mean radiation weighting factor, $\overline{w_{R}}$, values determined from International Commission on Radiological Protection (ICRP) Publication 92 (2003) and the calculated neutron spectral fluence in each organ. Risk of SMN was determined by calculating the excess relative risk $(E R R)$ in each organ using the linear no-threshold (LNT) model and organ-specific coefficients from the Biological Effects of Ionizing Radiation (BEIR) VII Committee (2006). For organs exposed to non-uniform doses, ERR was calculated voxel by voxel and the total $E R R$ for developing a SMN was computed as the sum of the $E R R$ values over all sensitive tissues (designated by $T$ ). This approach is mathematically equivalent to the formalism of Schneider et al (2007), who calculated absolute risk for organs irradiation with an inhomogeneous dose distribution. The $R R R$ value was then calculated as the ratio of the $E R R$ from proton therapy to the $E R R$ from photon therapy:

$$
R R R=\frac{E R R_{\text {proton }}}{E R R_{\mathrm{IMRT}}},
$$

where the $E R R$ for each modality $m$ is given by

$$
E R R_{\mathrm{m}}=\sum_{\mathrm{T}} E R R_{\mathrm{T}}
$$

where $E R R_{\mathrm{T}}$ is the $E R R$ of developing a second cancer in tissue $T$, and is given by

$$
E R R_{\mathrm{T}}=E R R_{1}+E R R_{2}=\left(D_{1, \mathrm{~T}} w_{1, R}+D_{2, \mathrm{~T}} w_{2, R}\right) \times R_{\mathrm{T}},
$$

where $E R R_{1}$ and $E R R_{2}$ are the $E R R$ of developing a second cancer in tissue $T$ from therapeutic and stray radiation, respectively; $D_{1, \mathrm{~T}}$ and $D_{2, \mathrm{~T}}$ are the absorbed doses to tissue $T$ from therapeutic and stray radiation, respectively; $\overline{w_{1, R}}$ and $\overline{w_{2, R}}$ are the mean radiation weighting factors of the therapeutic and stray radiation fields, respectively; and $R_{\mathrm{T}}$ is a tissue-specific risk coefficient. The calculated $R R R$ value for the 'medium' patient in our previous study was 0.66 (Fontenot et al 2009b), indicating that proton therapy confers a significantly lower risk of a SMN than IMRT. For the purpose of the present uncertainty analysis, the 'medium' patient, taken from a sample of ten consecutive patients from our clinic, is assumed to be representative of the patient population treated at our institution for low-risk prostate cancer.

\subsection{Uncertainties}

Using standard error propagation techniques, the uncertainty in $R R R$ can be expressed as

$$
\left(\frac{\sigma_{R R R}}{R R R}\right)^{2}=\sum_{\mathrm{m}}\left(\frac{\sum_{\mathrm{T}} \sigma_{E R R_{\mathrm{T}}}^{2}}{\left(\sum_{\mathrm{T}} E R R_{\mathrm{T}}\right)^{2}}\right)_{\mathrm{m}}-2 \frac{\sigma_{E R R_{\mathrm{h}} E R R_{\mathrm{P}}}^{2}}{E R R_{\mathrm{proton}} E R R_{\mathrm{IMRT}}},
$$

where the final term is the covariance. Expanding terms, the uncertainty in the $R R R$ (not including the covariance term) is 


$$
\begin{aligned}
& \left(\frac{\sigma_{R R R}}{R R R}\right)^{2} \\
& =\ldots \sum_{\mathrm{m}}\left(\frac{\sum_{\mathrm{T}}\left(E R R_{1}\right)^{2}\left(\frac{\sigma_{D_{1, \mathrm{~T}}}}{D_{1, \mathrm{~T}}}\right)^{2}+\left(E R R_{1}\right)^{2}\left(\frac{\sigma_{\overline{w_{1, R}}}}{\bar{w}_{1, R}}\right)^{2}+\left(E R R_{2}\right)^{2}\left(\frac{\sigma_{D_{2, \mathrm{~T}}}}{D_{2, \mathrm{~T}}}\right)^{2}+\left(E R R_{2}\right)^{2}\left(\frac{\sigma_{\overline{\bar{w}_{2, R}}}^{\bar{w}_{2, R}}}{{ }^{2}}\right)^{2}+\left(E R R_{\mathrm{T}}\right)^{2}\left(\frac{\sigma_{R_{\mathrm{T}}}}{R_{\mathrm{T}}}\right)^{2}}{\left(\sum_{\mathrm{T}} E R R_{\mathrm{T}}\right)^{2}}\right)
\end{aligned}
$$

The uncertainties associated with radiation weighting factors and risk models used in our previous work (Fontenot et al 2009b) are large and difficult to estimate from first principles. Therefore, we approximated $\sigma_{\overline{w_{R}}}$ and $\sigma_{R \mathrm{~T}}$ as zero for both modalities for the baseline $R R R$ calculations. The present analysis extends that work by taking into account plausible interval of values of $\sigma_{\overline{w_{R}}}$ and $\sigma_{R \mathrm{~T}}$. Additionally, we sought to characterize the uncertainty in the $R R R$ value arising from interpatient variations in organ doses, particularly to the rectum and bladder. This approach allowed for comprehensive testing of the sensitivity of the RRR to changes in these key variables, with the advantage of obviating the need for a detailed mechanistic understanding of the underlying processes of radiation carcinogenesis. Our approach to examining each of these variables is described below.

2.2.1. Mean radiation weighting factor-For the baseline $R R R$ calculation, we determined the $\overline{w_{R}}$ value used to convert absorbed dose, $D$, from neutrons to equivalent dose, $H$, using values recommended in ICRP Publication 92 and the calculated neutron spectral fluence. However, there is substantial uncertainty in $\overline{w_{R}}$ values. For example, the ICRP recommended a maximum neutron radiation weighting factor of 20 for low-energy neutrons, but a recent reanalysis of atomic bomb survivor data has suggested that the neutron RBE for carcinogenesis could be as high as 100 (Kellerer et al 2006). Therefore, we estimated the sensitivity of $R R R$ to uncertainties in the neutron radiation weighting factor by recalculating the $R R R$ value (using the LNT model) for the 'medium' patient using baseline $\overline{w_{R}}$ values that were scaled linearly by factors of 0.5 and 2 (see figure 1 ). These values were chosen because they bound the plausible interval of the true $\overline{w_{R}}$ value (Hall 2007).

Additionally, we re-computed with a larger scaling factor of 5 to determine the value of $\overline{w_{R}}$ at which $R R R$ exceeded unity (i.e. the point at which the risk of SMN from proton therapy was greater than that from photon therapy). Because neutrons are not generated from $6 \mathrm{MV}$ photon beams, we considered neutrons only in the case of proton therapy. The interval of $R R R$ values returned by different radiation weighting factors was used to estimate the $95 \%$ confidence interval of the $R R R$ value resulting from $\sigma_{\overline{w_{R}}}$.

2.2.2. Shape of the dose-risk model-During radiotherapy, patients are exposed to very low doses in some tissues and high doses in others. Risk data on SMN incidence in humans have been drawn mainly from studies of low-dose radiation, namely, exposures in survivors of atomic bombs (Preston et al 2003, 2004, Pierce et al 1996), radiation accidents (Hall 2006) and medical radiation procedures (Suit et al 2007). These data were also supplemented with results from animal and in vitro cell studies (Suit et al 2007). The consensus is that the dose-response curve is probably best estimated with the LNT model for exposures up to a few sieverts for most organs and tissues. As a result, the baseline $R R R$ 
value was computed on the assumption of an LNT relationship between exposure to radiation and risk of a SMN. However, at higher equivalent doses, available evidence suggests that significant deviations from the widely used LNT model are possible in some organs (Hall 2004).

Some studies of radiation-induced thyroid cancer in children have suggested a linear relationship between radiation exposure and carcinogenic risk up to approximately 20 Sieverts (Sv), with a decline in risk at doses higher than 15-20 Sv (Ronckers et al 2006, Sigurdson et al 2005). Meanwhile, studies of radiation-induced bladder cancer have indicated a linearly increasing relationship between exposure and carcinogenic risk at doses up to $10 \mathrm{~Sv}$, with a constant risk at higher doses (Brenner et al 2000, Boice et al 1988, Neugut et al 1997). Hence, two principal models have been derived that are believed to describe the shape of the dose-response curve at high equivalent doses: the linearexponential model (in which cancer risk increases linearly at low equivalent doses and then decreases exponentially at higher equivalent doses) and the linear-plateau model (in which the increase in risk is also linear at low equivalent doses and then levels off at higher equivalent doses) (Hall 2006).

We tested the sensitivity of the baseline $R R R$ values to uncertainties in the risk model by recalculating the $R R R$ using both the linear-exponential and linear-plateau models. Because we were modeling irradiation of the prostate, we needed to apply the linear-plateau and linear-exponential models only to the colon/rectum and bladder (because equivalent doses in all other tissues met the low-equivalent dose criterion of less than $2.5 \mathrm{~Sv}$ (Fontenot et al 2009a). Using the LNT risk models recommended in the BEIR-VII report, we added inflection doses to the risk model of each organ. Above each inflection dose, the two possible response curves (plateau and exponential decay) were modeled. The inflection doses were set at 10 and $40 \mathrm{~Sv}$, representing the extrema of plausible value reported in the literature. Thus, a total of four dose-response curves (two linear-plateau and two linearexponential curves) were tested for the 'medium' patient $R R R$ calculation.

The form of the risk models was similar to those suggested by Schneider et al $(2007,2006)$. The model describing a linear-exponential behavior of an organ-specific risk model, $R_{\mathrm{T}}$, is given by

$$
R_{\mathrm{T}}=R_{\mathrm{T}}^{0} H_{\mathrm{T}} \mathrm{e}^{-\alpha_{\mathrm{T}} H_{\mathrm{T}}}
$$

where $R_{\mathrm{T}}^{0}$ is the low-equivalent dose, organ-specific risk model from the BEIR-VII report, $H_{\mathrm{T}}$ is the equivalent dose to organ $T$ and $\mathrm{a}_{\mathrm{T}}$ is an organ-specific cell sterilization parameter. Similarly, the form of the linear-plateau model is given by

$$
R_{\mathrm{T}}=\frac{R_{\mathrm{T}}^{0}}{\delta_{\mathrm{T}}}\left(1-\mathrm{e}^{-\delta_{\mathrm{T}} H_{\mathrm{T}}}\right),
$$

where $R_{\mathrm{T}}^{0}$ and $H_{\mathrm{T}}$ are as previously defined and $\delta_{\mathrm{T}}$ is an organ-specific cell sterilization parameter. Numerical values for $a_{\mathrm{T}}$ and $\delta_{\mathrm{T}}$ for the colon/rectum and bladder were 
determined empirically. Additionally, we varied the $R_{\mathrm{T}}^{0}$ values slightly for each model so that the ERR value at $1 \mathrm{~Sv}$ would be consistent with the BEIR-VII value. Those parameters are listed in table 1.

2.2.3. Interpatient variations-We calculated the baseline $R R R$ value for a 'typical' patient. The machine settings required to deliver a prostate treatment to a particular patient are unique to the patient's anatomy. Therefore, we also examined the effects of anatomical and treatment variations that could influence the calculated $R R R$ value.

The effects of variations in therapeutic absorbed dose in the rectum and bladder across a population were assessed by determining the standard deviation of the absorbed dose in those organs in ten consecutively sampled patients who were treated for prostate cancer with proton therapy at our institution. For IMRT, the variation in stray absorbed dose was estimated from surveying monitor unit (MU) settings in treatment plans, following the method described by Fontenot et al (2008). For 6 MV photon IMRT, exposure to leakage radiation scaled directly with the MU setting. Thus, the predominant variable determining the amount of stray exposure in different patients was the number of delivered MUs.

The variation in stray absorbed dose from proton therapy was determined from Monte Carlo data previously reported by Fontenot et al (2008). In that study, proton beam range, spreadout Bragg peak width, collimated field size and air gap were varied over the interval of values used for prostate irradiation so that the sensitivity of stray absorbed dose to various clinical variables could be quantified.

\section{Results}

The results of the uncertainty analysis of each of the variables examined in this work are described below.

\subsection{Radiation weighting factor for neutrons}

The baseline $R R R$ calculation was somewhat sensitive to changes in $\overline{w_{R}}$, as can be seen in table 2. $E R R_{\mathrm{IMRT}}$ was not influenced by $\overline{w_{R}}$ because no neutrons are produced by $6 \mathrm{MV}$ photon beams. However, $E R R_{\text {proton }}$ did vary with $\overline{w_{R}}$. As $\overline{w_{R}}$ increased, the $E R R$ of organs out of the therapeutic field (for which stray equivalent dose was the only contributor to risk) increased linearly. Although neutron absorbed doses in the in-field organs (e.g. the rectum and bladder) were small, a large $\bar{w}_{R}$ for neutrons did significantly increase the $E R R$ in those organs. For example, the ERR in the bladder increased from 3.99 to $5.47(40 \%)$ when the $\overline{w_{R}}$ scaling factor was increased from 1 to 5. Overall, $R R R$ values for the scaling factors of $0.5,1$ and 2 were $0.61,0.66$ and 0.75 , respectively, suggesting that proton therapy can reduce the risk of SMN in patients undergoing radiation therapy for prostate cancer for plausible values of $\overline{w_{R}}$. When the scaling factor was set to 5 , the $R R R$ became greater than 1, indicating an elevated risk of SMN from proton therapy compared to photon therapy. However, a scaling factor of 5 would correspond to maximum $\overline{w_{R}}$ values of 100 for neutrons, which is not supported by the preponderance of current evidence (BEIR 2006, Newhauser et al 2009c). 


\subsection{Shape of the dose-risk model}

The baseline $R R R$ value showed only a small sensitivity to the shape of the dose-risk model. The calculated $R R R$ varied from 0.66 to 0.72 over all risk models examined (see figure 2). As expected, the $E R R_{\mathrm{T}}$ values in organs far from the therapeutic field were independent of the differences in the risk models since doses in those organs were below the roll-off doses. However, the $E R R_{\mathrm{T}}$ values in the bladder and colon/rectum showed substantial sensitivity to the selected risk model for both modalities. For the linear-exponential model with a $10 \mathrm{~Sv}$ roll-off, the in-field organs accounted for $26 \%$ and $76 \%$ of the total ERR for proton and photon therapies, respectively. For the linear-plateau model with a $40 \mathrm{~Sv}$ roll-off, the in-field organs accounted for $66 \%$ and $90 \%$ of the ERR for proton and photon therapies, respectively. In other words, when the risk from high equivalent doses was suppressed, the majority of the risk from the proton plans resulted from stray radiation. Conversely, the majority of the risk from the photon plans resulted from the therapeutic beam, independent of the risk model.

\subsection{Interpatient variations in anatomy and dose}

3.3.1. Therapeutic dose-The $R R R$ value was somewhat sensitive to anatomically related variations in exposure to the therapeutic beam. Based on an evaluation of ten patients (table 3), the mean therapeutic proton dose to the bladder and rectum varied by $\pm 62 \%$ and $\pm 23 \%$, respectively (95\% CI). For photon therapy, the mean equivalent dose to the bladder and rectum varied from the mean by $\pm 67 \%$ and $\pm 15 \%$, respectively ( $95 \% \mathrm{CI}$ ). Because exposure to the therapeutic radiation was the predominant contributor to $E R R$ in the baseline calculations, these variations in dose introduced enormous variations into the calculated $E R R$ values. However, for a given patient, dosimetric variations were highly correlated between the two modalities. Because variations in the mean dose to organs can principally be attributed to anatomical variations, if the anatomy of a given patient resulted in a large mean dose to an organ in a photon plan, then the same was true in the proton plan in nearly equal proportion. Thus, for any given prostate patient, there was strong positive covariance between the uncertainties in mean dose to the bladder and rectum. The dosimetric correlation coefficient $\left(r_{x y}\right)$ is used to characterize the strength and relationship of two variables $(x$ and $y)$ such that

$$
r_{x y}=\frac{\sum(x-\bar{x})(y-\bar{y})}{\sqrt{\sum(x-\bar{x})^{2} \sum(y-\bar{y})^{2}}} .
$$

Based on the data from this study, the value of $r$ between the mean bladder dose from proton and photon therapies was 0.95 . Similarly, the value of $r$ between the mean rectal dose from proton and photon therapies was 0.70 . Including these coefficients in the covariance term of equation (4) resulted in an uncertainty of $\pm 30 \%$ in the $R R R$ value resulting from interpatient variations in absorbed dose.

3.3.2. Stray radiation exposure-photon therapy-The $R R R$ value was not sensitive to variations in exposure to stray radiation from photon IMRT. Since leakage radiation during IMRT is proportional to the number of MU required to deliver the treatment, the variation in MU over a sample of prostate cancer patients provided an estimate of the 
variation in leakage dose from IMRT. In our sample of ten patients, the value of $95 \% \mathrm{CI}$ for MU was 124, or $17 \%$ of the mean MU value. However, the effect of this uncertainty on the $R R R$ calculation was small since exposure to leakage radiation represented only a small fraction of the $E R R_{\mathrm{p}}$. Thus, the uncertainty in absorbed dose from leakage introduced less than $1 \%$ uncertainty into the calculated $R R R$ values.

3.3.3. Stray radiation exposure-proton therapy-As reported in our previous work (Fontenot et al 2008), absorbed dose from stray neutron dose varied by about $50 \%$ over the interval of treatment parameters used for proton therapy of the prostate. However, as in the case of photon therapy, the effect of these dose variations on the $R R R$ calculation was small since exposure to stray radiation represented only a small fraction of the $E R R_{\text {proton. The }}$ uncertainty in absorbed dose from stray radiation introduced less than $5 \%$ uncertainty into the $R R R$.

\section{Discussion}

We conducted a systematic examination of uncertainties in the calculated $R R R$ of developing a SMN following contemporary proton to photon radiotherapies for cancer of the prostate. This revealed that the findings from our previous work-that passively scattered proton therapy could reduce the risk of SMN in early stage prostate cancer patients relative to photon IMRT - were relatively insensitive to the largest contributors to uncertainty in $R R R$. This insensitivity was mainly attributable to (1) large covariance terms (as was the case for the dose-response model and interpatient variations in absorbed dose) and (2) large relative uncertainties were associated with correspondingly small components of the ERR (as was the case with $\overline{w_{R}}$ ). Furthermore, while interpatient variations in absorbed dose had the greatest influence on $R R R$, uncertainties in patient-specific calculations of $R R R$ were quite small since dose distributions within a given patient are known with good accuracy.

Several recent reports have suggested that uncertainties in the value of $\overline{w_{R}}$ and possible nonlinearities in the dose-response model may prohibit meaningful comparisons of SMN risk between passively scattered proton therapy and photon IMRT (Hall 2007, 2009). However, our study indicates that relative risk analysis may be a valuable tool in performing such comparisons with acceptable levels of uncertainty. These results are consistent with previous studies from Newhauser et al (2009a), who demonstrated that uncertainties in $\overline{w_{R}}$ did not influence calculations of second cancer risk for pediatric patients receiving craniospinal irradiation with proton and photon therapies. Kry et al (2007) reported that relative risk analysis could provide meaningful risk assessment when the magnitude of uncertainties precluded absolute risk comparisons. Schneider et al (2008) also utilized relative risk analysis to compare different modalities, and found sensitivities to the shape of the dose-risk model similar to our data.

The uncertainty analysis presented in this work utilized a rigorous propagation of uncertainties in combination with sensitivity analysis. Beginning with highly accurate dosimetric data (Fontenot et al 2008, 2009b), the largest contributors to the uncertainty in calculated risk of SMN were identified and quantified. While it should be noted that the numerical findings of this study are not directly applicable to radiation treatments at other 
anatomical sites, the methodology is general and therefore is directly applicable to other sites with the appropriate adjustments of dosimetric data and risk models.

This work had several limitations. First, our one-dimensional analysis investigated the sensitivity of calculated $R R R$ values to uncertainties in parameters that were assumed to have a Gaussian distribution. The impact of multi-dimensional analysis (i.e. the interplay of simultaneous variations of several parameters) and non-Gaussian parameter distributions was not investigated. Further discussion of the impact of these assumptions is beyond the scope of this work, and the reader is instead referred to more theoretical texts (Saltelli et al 2008). Second, we used values of $w_{R}$ from report 92 of the ICRP. The ICRP has subsequently issued report 103 (2007), which provides updated values of $w_{R}$. However, due to the comparatively small changes in $w_{R}$ between reports 92 and 103, the major findings of this work would not be affected by using the most recent values of $w_{R}$. Finally, although calculations of $R R R$ provide useful comparative information regarding the risk of SMN, a more complete risk assessment would also include estimation of absolute risk for developing SMN. Although some epidemiological data are available, absolute risk data are highly uncertain and conclusions are controversial (Baxter et al 2005, Brenner et al 2000, Chrouser et al 2005, Kendal et al 2006, Neugut et al 1997, Pickles and Phillips 2002, SEER 2007) and, until the biological uncertainties described in this work are reduced to appropriately low levels, calculations of absolute risks of radiation-induced cancers will remain highly uncertain.

\section{Conclusions}

A systematic examination of uncertainties revealed that the calculated $R R R$ of developing a SMN following contemporary proton to photon radiotherapies for prostate cancer was insensitive to the largest contributors to the uncertainty. Uncertainties in the radiation weighting factor for neutrons, the shape of the dose-risk model and interpatient variations in therapeutic and stray doses introduced a $95 \%$ confidence interval of $\pm 33 \%$ around the nominal value.

\section{References}

Baxter NN, Tepper JE, Durham SB, Rothenberger DA, Virnig BA. Increased risk of rectal cancer after prostate radiation: a population-based study. Gastroenterology. 2005; 128:819-824. [PubMed: 15825064]

BEIR. Health Risks from Exposure to Low Levels of Ionizing Radiation BIER VII Phase 2. Washington, DC: National Research Council, National Academy of Science; 2006.

Boice JD Jr, et al. Radiation dose and second cancer risk in patients treated for cancer of the cervix. Radiat. Res. 1988; 116:3-55. [PubMed: 3186929]

Brenner DJ, Curtis RE, Hall EJ, Ron E. Second malignancies in prostate carcinoma patients after radiotherapy compared with surgery. Cancer. 2000; 88:398-406. [PubMed: 10640974]

Chrouser K, Leibovich B, Bergstralh E, Zincke H, Blute M. Bladder cancer risk following primary and adjuvant external beam radiation for prostate cancer. J. Urol. 2005; 174:107-110. discussion 10-1. [PubMed: 15947588]

Fontenot J, Taddei P, Zheng Y, Mirkovic D, Jordan T, Newhauser W. Equivalent dose and effective dose from stray radiation during passively scattered proton radiotherapy for prostate cancer. Phys. Med. Biol. 2008; 53:1677-1688. [PubMed: 18367796] 
Fontenot JD, Lee AK, Newhauser WD. Dose-rate effects when estimating risks for second malignancies. Int. J. Radiat. Oncol. Biol. Phys. 2009a; 75:1624-1625. [PubMed: 19931743]

Fontenot JD, Lee AK, Newhauser WD. Risk of secondary malignant neoplasms from proton therapy and intensity-modulated $\mathrm{x}$-ray therapy for early-stage prostate cancer. Int. J. Radiat. Oncol. Biol. Phys. 2009b; 74:616-622. [PubMed: 19427561]

Hall, EJ. Radiobiology for the Radiobiologist. Philadelphia, PA: Lippincott; 2006.

Hall EJ. The impact of protons on the incidence of second malignancies in radiotherapy. Technol. Cancer Res. Treat. 2007; 6:31-34. [PubMed: 17668949]

Hall EJ. Is there a place for quantitative risk assessment? J. Radiol. Prot. 2009; 29:A171-A184. [PubMed: 19454800]

Hall EJ. Henry S Kaplan Distinguished Scientist Award 2003. The crooked shall be made straight; dose-response relationships for carcinogenesis. Int. J. Radiat. Biol. 2004; 80:327-337. [PubMed: 15223765]

Howell RM, Hertel NE, Wang Z, Hutchinson J, Fullerton GD. Calculation of effective dose from measurements of secondary neutron spectra and scattered photon dose from dynamic MLC IMRT for $6 \mathrm{MV}, 15 \mathrm{MV}$, and $18 \mathrm{MV}$ beam energies. Med. Phys. 2006; 33:360-368. [PubMed: 16532941]

ICRP. ICRP. Vol. 33. Oxford: Pergamon; 2003. Relative biological effectiveness (RBE), quality factor (Q), and radiation weighting factor $(\mathrm{w}(\mathrm{R}))$; p. 1-117.

ICRP. ICRP. Vol. 103. Oxford: Pergamon; 2007. The 2007 recommendations of the ICRP; p. 1-332.

Kellerer AM, Ruhm W, Walsh L. Indications of the neutron effect contribution in the solid cancer data of the A-bomb survivors. Health Phys. 2006; 90:554-564. [PubMed: 16691103]

Kendal WS, Eapen L, Macrae R, Malone S, Nicholas G. Prostatic irradiation is not associated with any measurable increase in the risk of subsequent rectal cancer. Int. J. Radiat. Oncol. Biol. Phys. 2006; 65:661-668. [PubMed: 16545920]

Kry SF, Followill D, White RA, Stovall M, Kuban DA, Salehpour M. Uncertainty of calculated risk estimates for secondary malignancies after radiotherapy. Int. J. Radiat. Oncol. Biol. Phys. 2007; 68:1265-1271. [PubMed: 17637398]

Kry SF, Salehpour M, Followill DS, Stovall M, Kuban DA, White RA, Rosen II. Out-of-field photon and neutron dose equivalents from step-and-shoot intensity-modulated radiation therapy. Int. J. Radiat. Oncol. Biol. Phys. 2005; 62:1204-1216. [PubMed: 15990026]

Neugut AI, Ahsan H, Robinson E, Ennis RD. Bladder carcinoma and other second malignancies after radiotherapy for prostate carcinoma. Cancer. 1997; 79:1600-1604. [PubMed: 9118045]

Newhauser, W., et al. Contemporary proton therapy systems adequately protect patients from exposure to stray radiation. In: McDaniel, FD.; Doyle, BL., editors. 20th Int. Conf. on Application of Accelerators in Research and Industry; American Institute of Physics; Denton, TX. 2009a.

Newhauser W, Zheng Y, Taddei P, Mirkovic D, Fontenot J, Giebeler A, Zhang R, Titt U, Mohan R. Monte Carlo proton radiation therapy planning calculations. Trans. Am. Nucl. Soc. 2009b; 99:6364.

Newhauser WD, et al. The risk of developing a second cancer after receiving craniospinal proton irradiation. Phys. Med. Biol. 2009c; 54:2277-2291. [PubMed: 19305036]

Pickles T, Phillips N. The risk of second malignancy in men with prostate cancer treated with or without radiation in British Columbia, 1984-2000. Radiother. Oncol. 2002; 65:145-151. [PubMed: 12464442]

Pierce DA, Shimizu Y, Preston DL, Vaeth M, Mabuchi K, et al. Studies of the mortality of atomic bomb survivors. Report 12.1. Cancer: 1950-1990. Radiat. Res. 1996; 146:1-27. [PubMed: 8677290]

Preston DL, Pierce DA, Shimizu Y, Cullings HM, Fujita S, Funamoto S, Kodama K. Effect of recent changes in atomic bomb survivor dosimetry on cancer mortality risk estimates. Radiat. Res. 2004; 162:377-389. [PubMed: 15447045]

Preston DL, Shimizu Y, Pierce DA, Suyama A, Mabuchi K. Studies of mortality of atomic bomb survivors. Report 13. Solid cancer and noncancer disease mortality: 1950-1997. Radiat. Res. 2003; 160:381-407. [PubMed: 12968934] 
Ronckers CM, et al. Thyroid cancer in childhood cancer survivors: a detailed evaluation of radiation dose response and its modifiers. Radiat. Res. 2006; 166:618-628. [PubMed: 17007558]

Saltelli, A.; Ratto, M.; Andres, T.; Campolongo, F.; Cariboni, J.; Gatelli, D.; Saisana, M.; Tarantola, S. Global Sensitivity Analysis: The Primer. Chichester: Wiley; 2008.

Schneider U, Lomax A, Besserer J, Pemler P, Lombriser N, Kaser-Hotz B. The impact of dose escalation on secondary cancer risk after radiotherapy of prostate cancer. Int. J. Radiat. Oncol. Biol. Phys. 2007; 68:892-897. [PubMed: 17459608]

Schneider U, Lomax A, Pemler P, Besserer J, Ross D, Lombriser N, Kaser-Hotz B. The impact of IMRT and proton radiotherapy on secondary cancer incidence. Strahlenther. Onkol. 2006; 182:647-652. [PubMed: 17072522]

Schneider U, Lomax A, Timmermann B. Second cancers in children treated with modern radiotherapy techniques. Radiother. Oncol. 2008; 89:135-140. [PubMed: 18707783]

SEER. Surveillance Epidemiology and End Results (SEER) Program Populations (1969-2007). Bethesda, MD: National Cancer Institute; 2007.

Sigurdson AJ, et al. Primary thyroid cancer after a first tumour in childhood (the Childhood Cancer Survivor Study): a nested case-control study. Lancet. 2005; 365:2014-2023. [PubMed: 15950715]

Suit H, Goldberg S, Niemierko A, Ancukiewicz M, Hall E, Goitein M, Wong W, Paganetti H. Secondary carcinogenesis in patients treated with radiation: a review of data on radiation-induced cancers in human, non-human primate, canine and rodent subjects. Radiat. Res. 2007; 167:12-42. [PubMed: 17214511]

Taddei PJ, Fontenot JD, Zheng Y, Mirkovic D, Lee AK, Titt U, Newhauser WD. Reducing stray radiation dose to patients receiving passively scattered proton radiotherapy for prostate cancer. Phys. Med. Biol. 2008; 53:2131-2147. [PubMed: 18369278]

Taddei PJ, Mirkovic D, Fontenot JD, Giebeler A, Zheng Y, Kornguth D, Mohan R, Newhauser WD. Stray radiation dose and second cancer risk for a pediatric patient receiving craniospinal irradiation with proton beams. Phys. Med. Biol. 2009; 54:2259-2275. [PubMed: 19305045]

Zacharatou Jarlskog C, Lee C, Bolch WE, Xu XG, Paganetti H. Assessment of organ-specific neutron equivalent doses in proton therapy using computational whole-body age-dependent voxel phantoms. Phys. Med. Biol. 2008; 53:693-717. [PubMed: 18199910] 


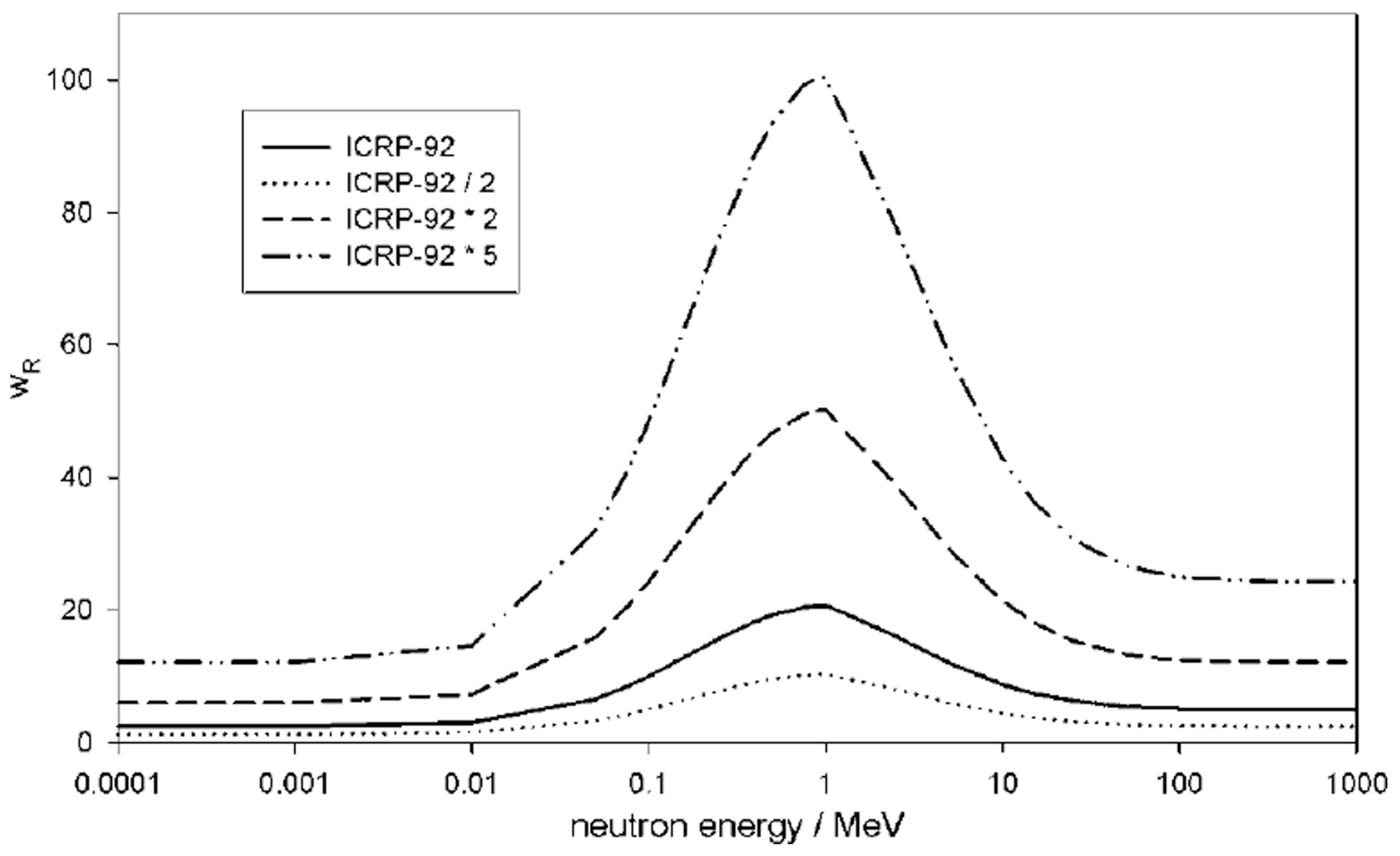

Figure 1.

Functions of the various radiation weighting factors $\left(w_{R}\right)$ for neutrons used to convert absorbed dose to equivalent dose from neutrons examined in this work. 


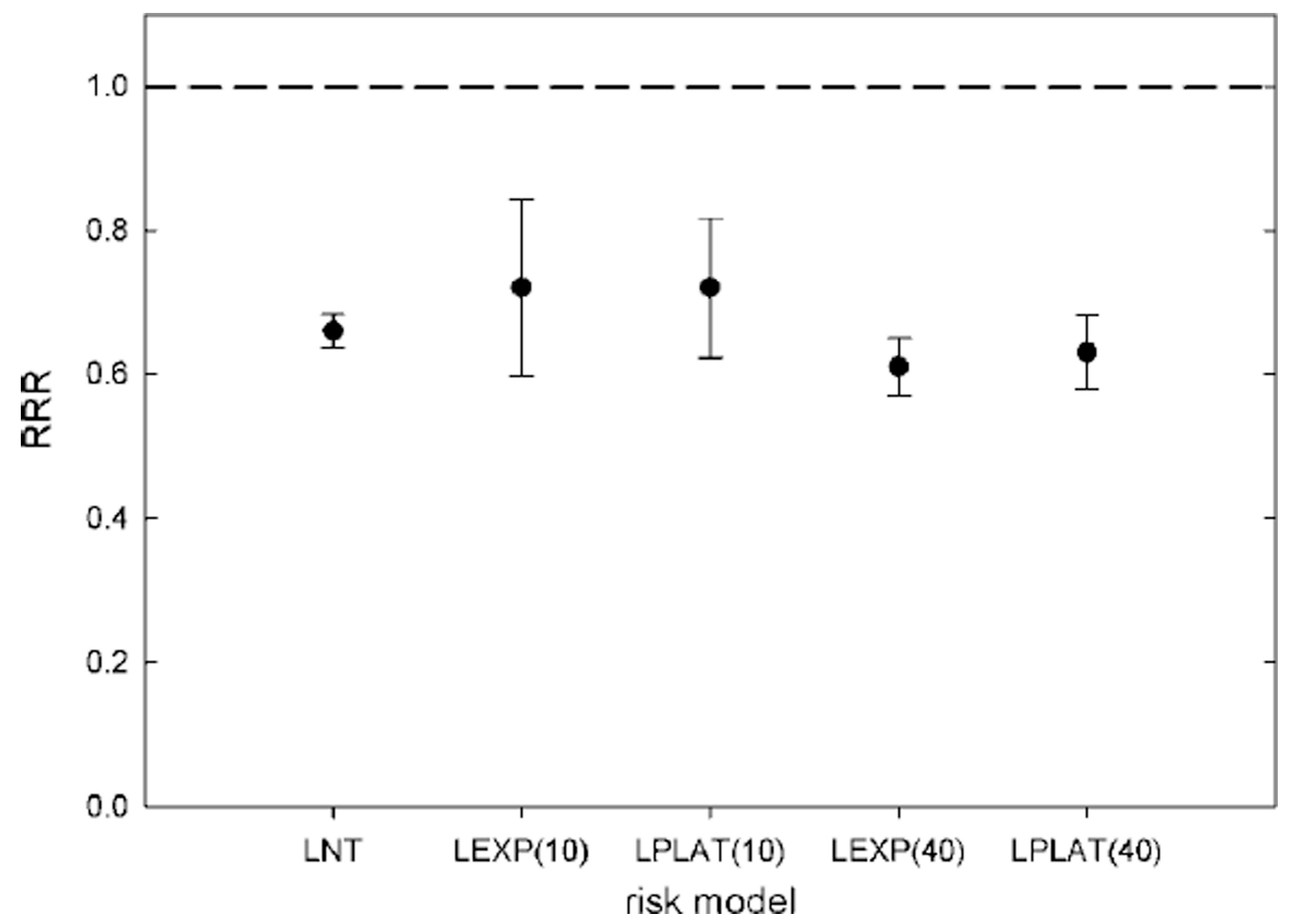

Figure 2.

The ratio of relative risk $(R R R)$, defined as the ratio of $E R R$ for protons $\left(E R R_{\text {proton }}\right)$ to that for intensity-modulated photon therapy $\left(E R R_{\mathrm{IMRT}}\right)$, of the medium patient for various doseresponse models. Error bars represent the uncertainty in $R R R$ due to variations in stray radiation exposures from proton therapy and IMRT in a population of ten prostate cancer patients. The response models were LNT, linear no-threshold; LEXP(10), linear-exponential rolling off at $10 \mathrm{~Sv}$; LPLAT(10), linear-plateau rolling off at $10 \mathrm{~Sv}$; LEXP(40), linearexponential rolling off at $40 \mathrm{~Sv}$; and LPLAT(40), linear-plateau rolling off at $40 \mathrm{~Sv}$. 


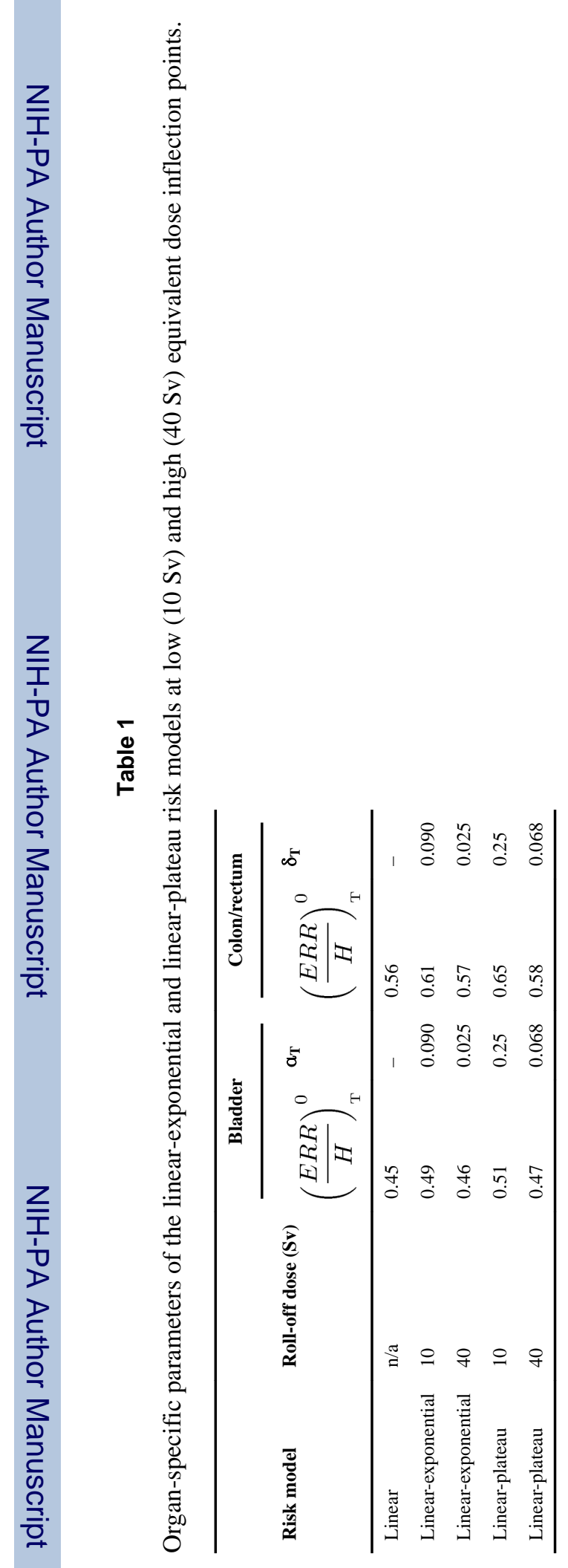




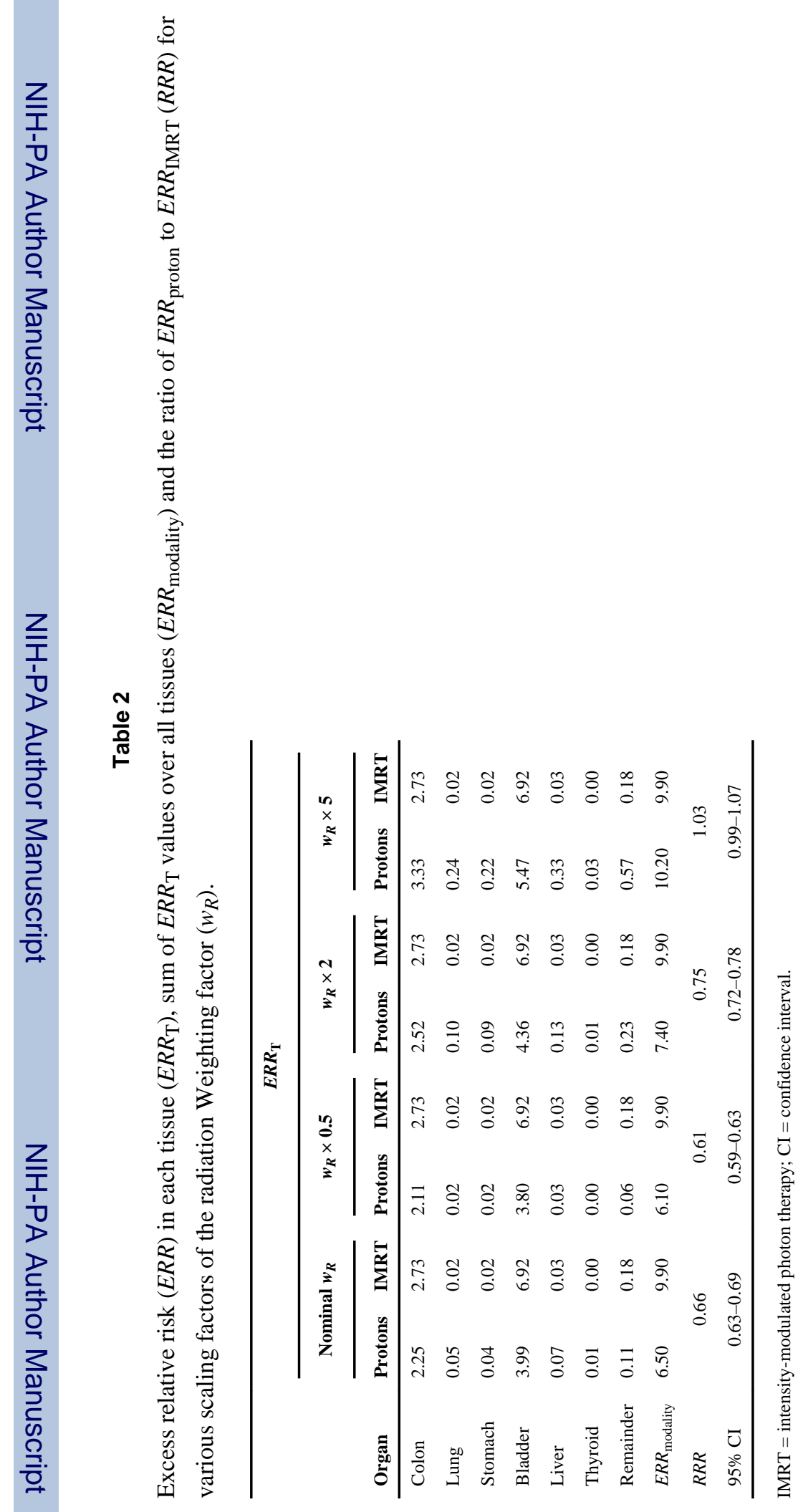


Table 3

Mean equivalent doses to the rectum and bladder from proton therapy and intensity-modulated radiation therapy (IMRT) of the prostate in ten patients.

\begin{tabular}{lrrrrr}
\hline & $\begin{array}{c}\text { Mean bladder } \\
\text { absorbed dose } \\
(\mathbf{G y})\end{array}$ & & \multicolumn{2}{c}{$\begin{array}{c}\text { Mean rectal } \\
\text { absorbed dose } \\
(\mathbf{G y})\end{array}$} \\
\cline { 2 - 3 } \cline { 6 - 6 } Patient number & Protons & IMRT & & Protons & IMRT \\
\hline 1 & 9.1 & 33.3 & & 19.4 & 34.9 \\
2 & 11.7 & 27.4 & & 22.7 & 38.9 \\
3 & 18.8 & 33.7 & & 20.4 & 35.4 \\
4 & 16.8 & 29.7 & & 21.7 & 31.6 \\
5 & 13.2 & 19.7 & & 21.4 & 33.8 \\
6 & 6.5 & 29.3 & & 26.9 & 34.2 \\
7 & 12.4 & 20.0 & & 24.1 & 37.6 \\
8 & 9.6 & 13.1 & & 22.5 & 35.3 \\
9 & 15.0 & 13.5 & & 22.0 & 33.5 \\
10 & 17.1 & 16.8 & 27.1 & 40.0 \\
Mean & 13.0 & 23.6 & 22.8 & 35.5 \\
SD $(1 \sigma)$ & 4.0 & 7.9 & 2.6 & 2.6 \\
\hline
\end{tabular}

$\mathrm{SD}=$ standard deviation. 